\title{
Model of Multi-Modal Cortical Processing: Coherent Learning in Self-Organizing Modules
}

\author{
Olivier Ménard \\ Supélec, Loria, France \\ E-mail: Olivier.Menard@ supelec.fr
}

\author{
Hervé Frezza-Buet \\ Supélec, France \\ E-mail: Herve.Frezza-Buet@supelec.fr
}

\begin{abstract}
In this paper $^{1}$, an original self-organizing model is presented, with experiments highlighting its ability to be used in different frameworks, as phonetic coding dependent on semantics and arm-reaching. The model relies on the coupling of the learning processes that stand at different self-organizing modules, and exhibits dynamics that can be discussed in terms of the binding of different modalities, scattered over the different modules. Such a binding property is based on an emerging constraint of keeping consistency between the modules. This process is induced by partial connectivity and appropriate neural field competition mechanisms.
\end{abstract}

\section{INTRODUCTION}

Biological studies of the brain have led to the design of artificial neural network models, promoting in the field of Computer Science the paradigm of fine grain interconnected numerical processing as a powerful method when adaptability and robustness are needed for numerical data. Some of these models are clearly oriented to explain biological observations, and are not suitable as efficient algorithm for computing data, in spite of their strong accuracy with regards to the biological data they are derived from. On the contrary, some other models rather express computational paradigms that seem to be involved in biological processes, but enhance computational efficiency, allowing the use of mechanisms that may be quite far from neuro-physiology, such as the explicit winner-takes-all in self-organizing maps (Kohonen, 1988). The model presented in this paper belongs to the latter group. It aims at instantiating a paradigm that comes from functional descriptions of cortical processings, mainly from early works as Burnod, 1989; Grossberg, 1976, but it follows computational guidelines that ensures relevance of the model in the field of Computer Science. Let us now discuss the two parts of that compromise.

The cortex, as described in biology, shows a capacity to spontaneously recruit neurons to adapt to dramatic changes of the information it receives: Visual cortex of the kitten is reorganized when it opens its second eye for example (Hubel, 1995). Moreover, it is able to organize any modality with very close mechanisms (see Miller, Pinto, \& Simons, 2001 for similarity between visual and somato-sensory cortex) by mapping action, perception, body, word semantics, and many

\footnotetext{
${ }^{1} \mathrm{An}$ abbreviated version of some portions of this article appeared in reference Menard \& Frezza-Buet, 2005, as part of the IJCNN 2005 conference proceedings, published under the IEEE copyright.
}

other information on a bi-dimensional neural structure. Seen as a whole, the cortex solves the contradiction of scattering specific computation dedicated to specific modalities over its surface, while having a quite homogeneous structure and keeping these computations coherent with each other.

The purpose of the model proposed in this paper is to exhibit such properties, while having computational features that make it feasible and innovative in the field of Computer Science. These features are the following ones. First, the computation is kept at the very level of the units, and does not require any external supervisory control. This allows efficient parallel implementation (Menard \& Frezza-Buet, 2004), and implies on-line learning, since no separated learning and test phases are allowed. Second, all plastic elements such as weights and activations, are initialized randomly. This prevents the processes from being dependent on some particular starting state. Third, all the units involved in the model are numerical and have the same computation rules: they specialize according to the connectivity and the differences in external inputs they receive.

Such an approach originates from preliminary works concerning the modeling of a generic computational unit (Alexandre, Guyot, Haton, \& Burnod, 1991), that have been extended in Frezza-Buet \& Alexandre, 2002 to deal with goal-oriented processes and strictly local cellular automata.

\section{THE BIJAMA MODEL}

The model we have developed is called Biologically Inspired Joint Associative Maps (Bijama). The general features of this model are presented in the following paragraphs, highlighting its dynamics and its properties, before the description of two different uses of that model in the next sections.

\section{A. Cortical maps and unit computation}

Our model organization is inspired from that of the biological cortex, more specifically on its modeling in terms of cortical maps, as described in Burnod, 1989. Cortical maps, in our model, are composed of computational units that tile a 2D sheet. In each map, the units are all related to the same modality of perception or action. A Bijama implementation of a specific sensory-motor task consists of one cortical map for each modality implied in the task, and some supplementary cortical maps, rather associative, that relate these modalities together. 
Within a map, units perform three computational processes. These processes are only briefly presented here, as they will be detailed in section 2-C. First, each unit computes a matching activity $A^{g}$ that represents its recognition of the current configuration of its inputs. The computational details of $A^{g}$ will be discussed later, let us note here that a unit with high $A^{g}$ fits the current inputs configuration. Second, in order to process a decision, small patches of units must be chosen in each modality. As the noisy pattern of $A^{g}$ is unsuitable for this, units perform a local competition process based on $A^{g}$ within each map, which result in another activity called $A^{\star}$. This competition process is derived from the theoretical results of the Continuous Neural Field Theory (CNFT) (see. Amari, 1977; Taylor, 1997). The $A^{\star}$ activities are related to the $A^{g}$ activities they are built from. Units with non-null $A^{\star}$ activity form some compact isolated groups, usually called "bubbles" of activity in the neural field literature. $A^{\star}$ bubbles of activity stand where $A^{g}$ activities are the most significant (cf. fig. 1), and can be considered as a focusing process that is the basis of a distributed decision mechanism.

Third, a main computational process at the level of the units is a learning mechanism that is roughly similar to that present in Kohonen's Self-Organizing Maps (S.O.Ms). Units in the $A^{\star}$ activity bubbles learn to better fit their current configuration of inputs. However, a dramatical difference between Bijama and SOMs is that some of the inputs to a unit in one map are the $A^{\star}$ output from units in another map. Therefore, the self-organization processes in connected maps are actually interdependent. This linkage between cortical maps is central to the Bijama model and will be discussed now.

\section{B. Inter-map stripe connectivity}

As presented in section 2-A, our model scatters related information in different cortical maps. In order to process this information, the cortical maps are linked together, so that units in a map have access to the information processed in other maps. When two maps are connected, however, a unit in a map doesn't receive information from all the units in the other map. Instead, it receives information from a stripe of units in the remote map (cf. fig. 2). All stripes from a given remote map have the same direction. It will be detailed in the next paragraph that this inter-map connectivity is what allows our model to address the binding problem: Information is processed locally within a map, but the local processing tasks communicate with each other through the connection stripes to form a coherent whole.

This limited connectivity is biologically inspired, as it resembles the connections between different cortical areas mentioned in Burnod, 1989. It also has a computational purpose, since a full connectivity leads to a combinatorial explosion as the size and number of the cortical maps grow. Since this model has been designed to handle complex tasks requiring multiple cortical maps, the map-to-map connectivity has to be limited.

When a map is connected to several remote maps, its units receive input from several stripes, one stripe per remote map.
For each cortical stripe $S_{i}$ to a unit, a cortical activity $A_{i}^{c}$ is computed. $A_{i}^{c}$ represents the fitting of the actual cortical input (i.e. the remote $A^{\star}$ activities from the stripe) with the preferred cortical input of the unit from that very stripe (cf. section 2-C). These $A_{i}^{c}$ activities are of primary importance in the computation of $A^{g}$. As detailed in section 2-C, $A^{g}$ is dependent on the product of the $A_{i}^{c}$ s. So a high $A^{g}$ for a unit $u$, and as a consequence the rising of a $A^{\star}$ bubble at that place, requires that remote $A^{\star}$ bubbles actually stand in the stripes intersecting at $u$ (see figure 1). So the model is implicitly controlled by an underlying relaxation process that tends to keep bubbles of $A^{\star}$ at the intersection of connection stripes.

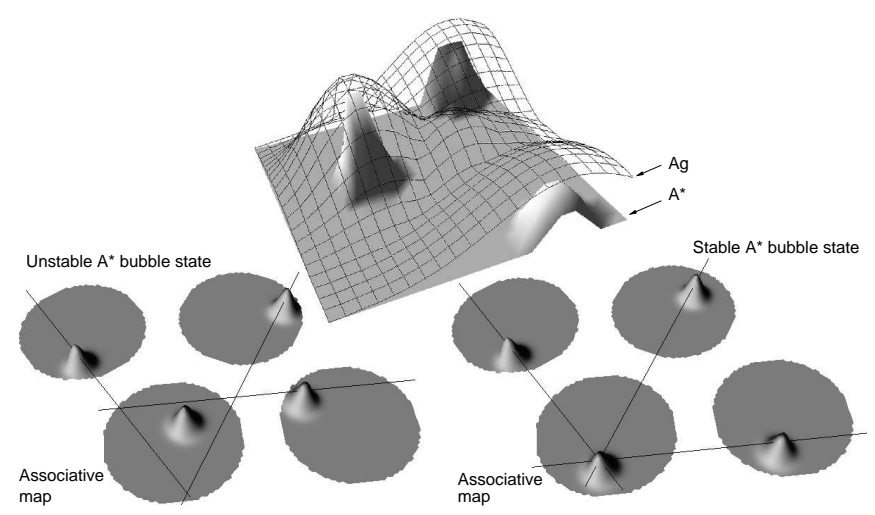

Fig. 1. Conditions for stable $A^{\star}$ activity states. On the left, unstable state where bubbles of $A^{\star}$ don't stand at intersecting stripes. On the right, stable state: Any other bubble position in the same stripes on the three nonassociative maps would also be stable. Top part shows the $A^{\star}$ activity bubbles formed from $A^{g}$ activity. An $A^{\star}$ activity bubble appears where the $A^{g}$ activity is the strongest in its neighborhood.

This stabilization of activity occurs when maps are connected reciprocally. That means that when a map is fed with stripes following a direction, it feeds back the remote map with reciprocal stripes in the same direction. The resulting dynamics can be compared with the phenomenon of resonance described in the ART paradigm by Grossberg, 1976, and produces stable and coherent states across the different maps. It ensures consistency of the activity bubbles across many connected maps by keeping $A^{\star}$ bubbles connected together, in spite of the partial connectivity induced by the stripes. This is of primary importance for learning. The model stresses that the learning rate of units is modulated by their $A^{\star}$. Thus, units whose $A^{\star}$ are activated simultaneously in the different maps at stable places learn together. We call this coherent learning. Learning strengthens the connections, that actually exist for stable states, between these coherent units. By this mechanism, they will tend to activate together again in the future, and then make related information on the maps be learned by actually connected units.

The central point of our model is that learning within a map depends on some other maps, so that the inter-map connectivity drives the convergence to a particular self-organized state, when self-organization alone would have allowed for many more possible ones. The final state of organization in 
each map must allow the bubbles to be set up at intersecting cortical connection stripes, solving inter-map constraints as the one illustrated on fig 1 . The cortical maps perform an effective compromise between the local and remote constraints. Remote constraints, coming from the architecture, make activity bubbles have strong cortical connections to each other. As presented later (cf. section 2-C), the maps are also under specific constraints, depending of the external perceptual input they receive. These constraints tend to make bubbles rise at places where units fit best the current external input. At first, the compromise between this input-fitting on the one hand, and the inter-map stability mentioned before on the other hand, is quite poor. However, and this is the main result of the model dynamics, this compromise gets better as learning proceeds.

To conclude, the combination of self-organization and coherent learning produces what we call joint organization: Competition, although locally computed, occurs not only inside any given map, but across all maps. Moreover, the use of connection stripes limits the connectivity, which avoids the combinatorial explosion that would occur if the model were to employ full connectivity between the maps. Thus, coherent learning leads to both efficient data representation within each map and coordination between all connected maps.

\section{Matching activities and prototype learning}

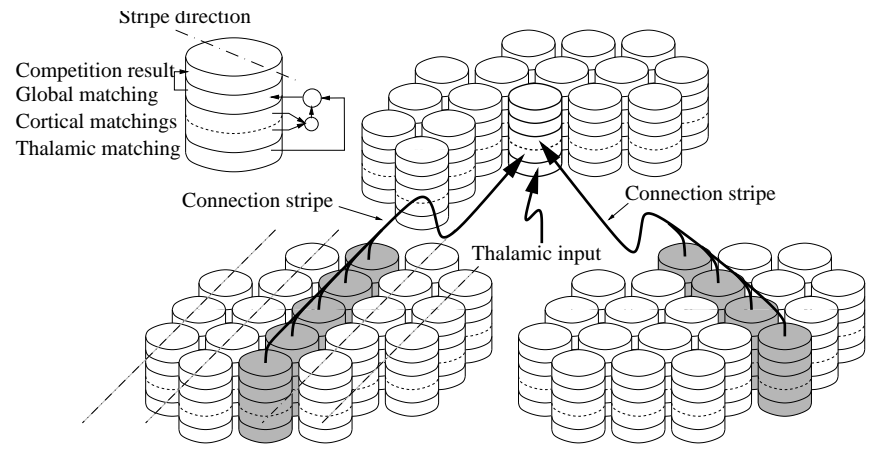

Fig. 2. The bold unit is connected to two stripes of remote units, and has a thalamic input. It therefore computes a thalamic matching activity and two cortical matching activities, and merges them together to from the $A^{g}$ activity. The $A^{\star}$ activity is obtained form the local competition based on $A^{g}$.

In section 2-A, it was stated that Bijama units compute a matching $A^{g}$ activity that represents how well they fit their configuration of inputs. As a unit receives different types, of inputs, this activity is actually formed as the merging of basic matching activities. Each of these basic merging activities is related to a specific input type the unit receives. For each of these input types, the unit compares the current input configuration with an internal prototype. This prototype is the input configuration the unit recognizes best. The matching activity follows a Gaussian tuning curve, and gets weaker as the input gets different from the prototype.

Basic matching activities can be classified into two types: thalamic activities and cortical ones. Thalamic activities correspond to the inputs coming from outside the Bijama cortical model: typically, they are sensory inputs. They are so called since they refer to the input the cortex receives from the thalamus, but the model implements a very functional view of that point. A thalamic input is a vector $I=\left(i_{k}\right)_{1 \leq k \leq n}$, and the corresponding prototype is a vector $P$. The matching activity $A^{\theta}$ and the associated learning rule are given in equation 1 , where $\alpha_{\theta}$ and $\delta_{\theta}$ are constants dependent on the actual thalamic input type.

$$
A^{\theta}=\exp \left(-\alpha_{\theta} \sum_{k}\left(i_{k}-p_{k}\right)^{2}\right), \Delta P=\delta_{\theta} A^{\star}(I-P)
$$

In the learning process, units in the $A^{\star}$ activity bubble modify their thalamic prototype so that it becomes closer to the thalamic input.

As detailed in section 2-B, units receive input from other cortical maps. These inputs are used to compute cortical matching activities $A_{i}^{c}$ s, that represent the detection of a configuration of a remote $A^{\star}$ activity pattern from a stripe connected to the unit. Units compute one cortical matching activity per stripe they are connected to. A cortical activity $A_{i}^{c}$ is computed from the remote activities $A_{k}^{\star}$ in the stripe, and the corresponding prototype vector $W$ according to equation 2 . This equation also presents the cortical learning process, that is based on the Widrow-Hoff learning rule, for reasons that will be explained in section 3-B. $\epsilon$ is a leak parameter.

$$
A_{i}^{c}=\sum_{k} w_{k} A_{k}^{\star}, \Delta w_{j}=\left(\delta A_{i}^{\star}+\epsilon\right)\left(A_{i}^{\star}-\sum_{k} w_{k} A_{k}^{\star}\right) A_{j}^{\star}
$$

The cortical activities are then merged to form a "cortical pattern recognition" activity $A^{c}=\sqrt[n]{\prod_{i=1}^{n}\left(A_{i}^{c}+\beta\right)}-\beta$ that is some kind of fuzzy "and" between the $A_{i}^{c}$ s activities. $A^{c}$ is then merged with $A^{\theta}$ (if it exists) to form $A^{g}=$ $\sqrt{A^{\theta}\left(A^{c}+\eta\right)}$, where $\eta$ ensures that $A^{g}$ is not null on the whole map even in the absence of cortical recognition.

\section{Competition process}

As detailed in section 2-A, the $A^{g}$ activity is the basis for a competition process that is local to the map. The competition results in activity $A^{\star}$, which forms bubbles of activity within a map. This competition process uses both short-range excitatory connections and long-range inhibitory ones. It is inspired from other on-center off-surround competition mechanisms, which can be found in Amari, 1977; Taylor, 1997.

However, a drawback of these previous mechanisms is that they rely on the sum of lateral influences for competition. This makes the mechanism very sensitive to the actual number of connections to a unit, both excitatory and inhibitory. More precisely, these mechanisms provide satisfying results when all units in a map have the same lateral connection profile. This forces the use of an architecture without borders for the cortical maps (a toroidal one for instance). Units on the border of the maps would otherwise have different dynamics, since they don't have a connection profile similar to that of units in the center of the map. To sum up, when a map has borders, dramatic side effects appear, as bubbles of $A^{\star}$ will only arise in the corners of the map whatever the inputs are to the map. 
In order to avoid this drawback and allow maps with any shapes (we use round ones in the model), the competition mechanism must be made independent of the number of connections to a unit. This is the reason why we choose not to rely on the sum of lateral influences but on the maxima of these influences, whose values are independent of the number of connections. We are unfortunately unable to provide formal proof that the following mechanism, based on maxima and non-linearities, produces bubbles of $A^{\star}$ activity, but it does so very reliably in our experiments.

Let us now detail the competition process occurring at unit $i$. Let $\omega_{i l}^{+}=\exp \left(-(i-l)^{2} / \sigma_{+}^{2}\right)\left(\right.$ resp. $\left.\omega_{i l}^{-}=\exp \left(-(i-l)^{2} / \sigma_{-}^{2}\right)\right)$ be the excitatory (resp. inhibitory) connection weights from units $l$ to unit $i$. The $\omega^{+}$and the $\omega^{-}$each form a positive Gaussian distribution centered on $i$. Let $f$ [.] be a sigmoidtype non-linearity. At each time step, the model computes the $n^{+}$top values of $\omega_{i l}^{+} \times A_{l}^{\star}$ and the $n^{-}$top values of $\omega_{i l}^{-} \times A_{l}^{\star}$. Let the sum of these maximal values be respectively inf ${ }^{+}$and inf $^{-}$. The use of inf ${ }^{+}$and inf $^{-}$as lateral influence in the competition process, instead of the sum of all contributions, ensures that some side-effects of the competition mechanism related to the actual number of connections are avoided. At each time step, $A_{i}^{\star}$ is updated according to the following rule, where $\rho, \phi, \tau, v$ are positive constants:

$$
\Delta A_{i}^{\star}=\delta_{\star}\left(f\left[\rho \inf ^{+}-\phi \inf ^{-}+\tau A_{i}^{g}-v A_{i}^{\star}\right]\right)
$$

This mechanism allows the formation of bubbles at locally best matching places (see. fig. 1) as the CNFT does.

Let us now consider two uses of this model, as in Menard \& Frezza-Buet, 2005; first a word/action association problem, that exhibits joint organization properties and then a visually guided arm reaching task that highlights the need of rewarding signals in motor learning with such a model.

\section{THE WORD-ACTION ASSOCIATION PROBLEM}

We have applied our model to a simple word-action association task, as part of the European Mirrorbot project. A more detailed explanation of this problem can be found in Menard, Frezza-Buet, \& Alexandre, 2004.

Several brain imaging studies as Pulvermüller, 2003 have shown that word encoding within the brain is not only organized around purely phonetic codes but is also organized around action. How this is done within the brain has not yet been fully explained but we would like to present how these action based representations naturally emerge in our model by virtue of solving constraints coming from motor maps.

A part of the set of words used in the MirrorBot project was used in a "phonetic" map, and we have tried to associate these words to the body parts that perform the corresponding action. Each word is semantically associated to a specific actuator. The word-actuator relationship is shown on figure 3 .

\section{A. Model architecture}

Here, three cortical maps are used: One for phonetic words representation, one for action representation and finally an associative one that links words to actions (cf. figure 3). Units

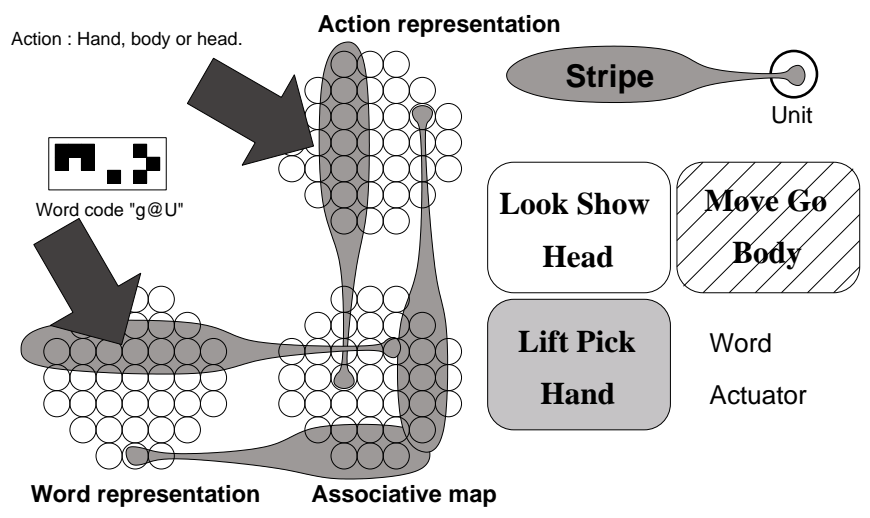

Fig. 3. Schematic view of the model architecture: The word representations and the action representations are presented in separate maps, that are both connected to an associative map by reciprocal cortical connection stripes. On the right, words-actuator relation is presented.

in the "word" map receive as thalamic input a phonetic coding of a word while units in the "action" map receive as thalamic input the "action" of a body part (head, body or hand), which corresponds to the word. Units in the associative map receive no thalamic input.

\section{B. Multi-association and cortical learning rules}

This association task highlights a problem that arises whenever two modalities must be associated. The associations between instances of the two modalities (here between phonetic words and actions) can be not only one-to-one, but also oneto-many, or even many-to-many. This is a multi-association problem. Here, each "action" is associated with two words.

In our experiments, if we want to use only one unit to represent each action, that unit must then be strongly connected to the phonetic representations of two words (cf. fig. 3-right). Thus, a single unit in the associative map must bind together one unit in the action map and two units in the word map. If an associative unit cannot be strongly linked to more than one word unit, then each action must be represented by two different units in the action map, and each of these units must be bound to a single word.

Such a duplication is highly undesirable, as it means that each time a new association is made, a new unit must be recruited by the self-organizing process. As the number of units should stay as small as possible, it is mandatory for associative units to have the multi-association ability: A single associative unit must be able to be strongly connected to many remote units in the same cortical stripe. It is actually the cortical learning rule that enables, or not, a unit to have this multi-association ability. Therefore, it is the key for solving the multi-association problem.

Let us now consider the Hebb/anti-Hebb learning rule. Using this rule, if unit $i$ is connected to a stripe of remote units $j$, the global connection strength of the local unit $i$ is at every time distributed among all connected units $j$ in the stripe. Since that connection strength must be high for some connection, it is concentrated on a single remote unit, which 
means that all other remote units are very weakly connected to the local unit. This is the reason why, using the Hebb/antiHebb learning rule, a unit $i$ can be activated by a unit $j$ if an $A^{\star}$ bubble around $j$ is the only one that is correlated to $A_{i}^{\star}$. The end result is that the local unit reacts to the $A^{\star}$ activation of a single remote unit per stripe connection.

As a consequence, the model cannot represent a situation where a unit in a map should be bound to multiple units in the other remote maps: It cannot handle multi-associations.

This can be avoided if the cortical learning rule allows a unit to be strongly associated with multiple units for each cortical stripe. A cortical learning rule that allows this is the WidrowHoff learning rule (cf. eq. 2). Here, the global connection strength of a local unit $i$ for a given cortical stripe is distributed among all currently active remote units $j$, and not among all remote units. As with the Hebb/anti-Hebb rule, because of the local competition, the connection strength $w_{i j}$ between $i$ and a unit $j$ must be high. However, here, increasing $w_{i j}$ doesn't imply decreasing all $w_{i k}$ for all $k$ in the remote connection stripe. Increasing $w_{i j}$ will only lower $w_{i k}$ if $j$ and $k$ are active at the same time.

When only a small $A^{\star}$ activity bubble is present on each map, only few remote units in a given connection stripe can be active at the same time. Thus, the local unit $i$ can react to the $A^{\star}$ activation of multiple units in a given stripe connection to a unit in another stripe connection. This is the reason why the Widrow-Hoff learning rule is used in the Bijama model.

\section{Results}
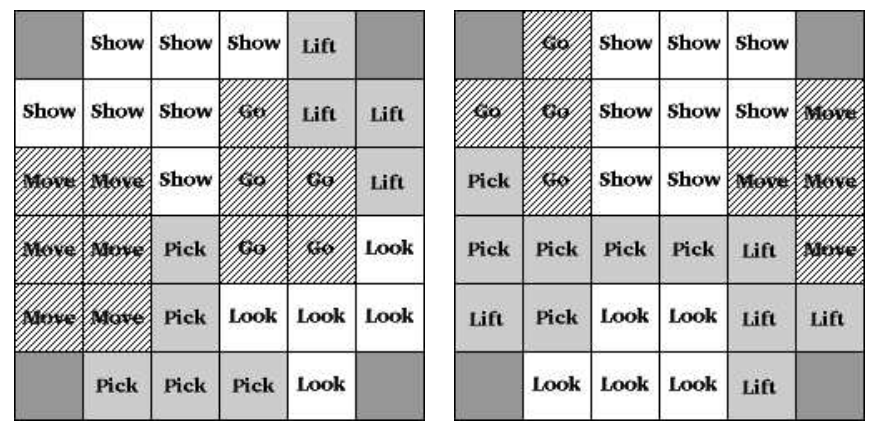

Fig. 4. Two different results of word classification by a Kohonen map based on purely phonetic representations. Words representing head action (white), hand action (gray) or body action (stripes) are spread all over the map without paying any attention to the underlying semantics of words.

One of the main interests of this word-action association task is to show how the joint organization provided by the Bijama model differs from a classical Kohonen organization. If we were to use a Kohonen map to represent words from the MirrorBot grammar (encoded as a phonetic sequence), a consequence of the Kohonen algorithm and existing lateral interaction between units would be an organization toward similarity relation of word codes only (i.e. two words having similar code would be represented by the same prototype or neighbor prototypes) as illustrated in figure 4 . This kind of representation is not suitable in the sense that it is totally disconnected from other maps and does not take any semantics of words into account.
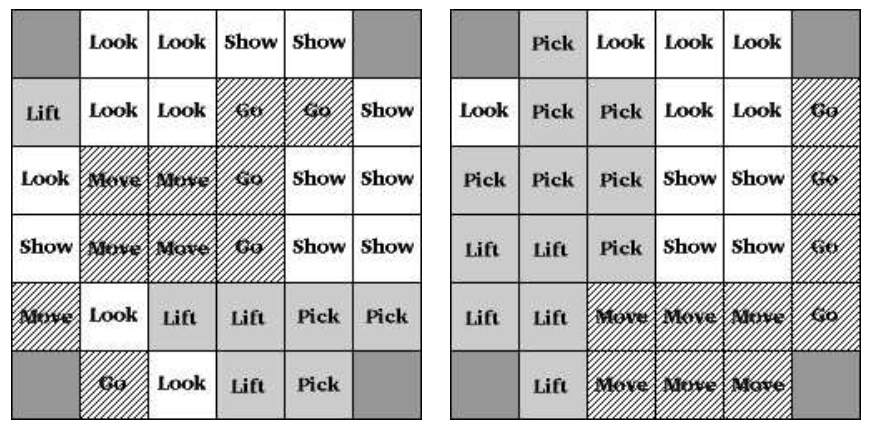

Fig. 5. Two simulation results of word representation map after coherent learning has occurred with our model. Word representations are now constrained by the motor map through the associative map and, as a result, words that correspond to the same action are grouped together. Nevertheless, phonetic proximity is still kept.

Within the word map, as illustrated in figure 5, words are grouped relative to the body part they represent: Body action words are grouped together (stripes) as well as hand action words (gray) and head action words (white).

However, the phonetic distribution of words remains the most important factor in the phonetic map organization. Each word is represented by a "cluster" of close units, and the words whose phonetic representation is close tend to be represented in close clusters of units. For instance, while "Go" and "Show" correspond to different motor actions, their phonetic representations are close, so that their representing clusters are adjacent (cf. fig. 5). This illustrates the fact that the model is actually doing a successful compromise between the local demands, which tend to organize the words phonetically, and the motor demands, which tend to put together the words that correspond to the same action. The joint organization does not destroy the local self-organization, but rather drives it so that it becomes coherent with the other map organization.

\section{VISUALLY GUIDED ARM REACHING TASK}

Another application of the Bijama model is coordinate transformation, such as the one the cortex performs in sensorymotor tasks (Guigon, 2003). More precisely, the example presented here is the visually guided reaching movement of an arm. The task consists of guiding one's hand towards a target while seeing both the hand and the target. We use here a simplified version of the problem, as it does not deal with $3 \mathrm{D}$ vision and the arm only has two degrees of freedom.

The Bijama model, here, has two perceptual inputs: vision and arm posture (proprioception). The visual component is handled as the relative position of the target from the hand in a Cartesian visual frame reference. The arm posture is given as the combination of the shoulder and elbow angles, and is thus expressed in a muscle frame of reference. The action performed by the model is a movement of the arm, considered as a change in the arm posture, and is expressed in the same frame of reference as posture is. In order to 
perform this task efficiently, the visual and the arm muscle frames of reference must both be used: Depending on the arm posture, a single visual demand can be achieved through very different arm movements. Thus, the model must relate these two frames of reference. A variation of this task has already been studied with success, by using S.O.Ms (see Martinez, Ritter, \& Schulten, 1990), but dealing with absolute visual information and thus implementing the learning of a one-to-one correspondence between visual position and proprioception.

The Bijama model, as presented to this point, is not able to accomplish this task: Here, the model would have to perform a motor action, chosen as the prototype of a unit in the motor map. However, as detailed in section 4-B, the motor actions cannot be considered in the same way as thalamic inputs, as this would require knowing the correct motor action beforehand, to train the model with. It has been shown in Menard \& Frezza-Buet, 2003 that the Bijama model can learn the sensory-motor association problem expressed here, when provided with coherent triples of visual target, posture and motion to perform. Providing such data to train the model requires knowing the inverse model of the arm. For computer science purposes, this is not acceptable since having the inverse arm model at disposal, for the generation of the training data, is sufficient for arm control, without requiring any supplementary architecture. Some models can afford to have the inverse model available for the setting-up of training data, as in Guigon, 2003, since their very purpose is to analyze the existence of biologically plausible activations once the neural network is trained. As our model is much more functional, it is only given the data acquired during its own interactions with the external world. This restriction has made the concept of rewarded actions naturally enter our approach. Our solution, that avoids the use of an inverse model, is to compute the correctness of the actions spontaneously triggered by the architecture, and to reward efficient ones. This reward computation roughly refers to the role of reward signals in the basal ganglia of the mammalian brain (see Doya, 1999). The structures that provide this rewarded learning are presented in the next section.

\section{A. Model architecture}

In this application of the Bijama model, two perceptual maps (one visual and the other proprioceptive), a motor map and an associative map, that links the three other maps together, are used. The model architecture is depicted in fig. 6 . The visual input is a $(x, y)$ vector represents the position of the target relative to that of the hand. The proprioceptive input $\left(\theta_{S}, \theta_{E}\right)$ represents the arm posture by the shoulder and elbow angles. The motor output $\left(\delta \theta_{S}, \delta \theta_{E}\right)$ is a variation of them.

New modules have been added to the Bijama model in order to compute and handle rewarded learning. One of these is the so-called spinal map. This map is connected to the motor map, and its active units (in $A^{\star}$ terms) are those that correspond to the action performed. The activation rule in the spinal map is sensitive to the reward, as explained in the next section. Each spinal map unit corresponds to a specific hard-wired movement that is not learned. The other new feature is the so-called basal unit. Role of this unit is to predict reward from the cortical map $A^{\star}$ activity patterns, and to forward this prediction to all spinal units. A more detailed look at these structures can be found in Menard et al., 2004. This framework aims at testing the Bijama model capabilities, rather than accurately modeling the motor cortex, the spinal cord and the basal ganglia.

\section{B. Rewarded learning}

Unsupervised learning, as used in S.O.Ms, would seem a natural choice for the Bijama model. However, as we will explain now, it is not easily capable of handling sensorymotor skills. While the following explanation is focused on the visually guided arm reaching task, its arguments hold true for many servo-control learning tasks in the cortex.

Moving the hand towards a visual target implies transforming a goal in the visual space into a muscle activity, while considering the current arm posture. Learning this task, as shown by the biological model by Guigon, 2003, implies finding a variation of muscle position that will move the hand towards the target for every possible target position and arm posture. A basic learning idea is then to generate random arm movements (motor babbling), and to determine which targets could be reached by these movements in the current arm posture. A self-organizing process would then lead to a complete mapping of reachable visual positions, given the arm posture. By inverting this model, one could seemingly choose a movement that would change the arm posture so that the hand reaches the target.

However, such an approach has a fatal drawback: the distribution of visual targets generated in this manner is not uniform in the visual space. Thus since the usual unsupervised techniques are very sensitive to the input distribution density, certain visual positions would become unreachable, because too few movements have reached them while learning took place. Since every target position must be reachable, a solution would be to teach the model by using random target positions and arm postures. However, that requires using an inverse model of the reaching problem in order to select the correct arm movement. As mentioned before, it is not admissible for a computer science model that focuses on action selection. It is especially true when, as is the case here, the model should be able to address problems for which the inverse model is not available or ambiguous because of redundancy. Therefore, the Bijama model must use another way to ensure that welldistributed visual examples are used.

The Bijama model uses rewarded learning in order to ensure both a correct distribution of sensory prototypes in the sensory maps, and the learning of efficient motor actions (arm movements). The functional principle of the model is as follows: a random target position and arm posture are first generated. These serve as sensory inputs to the model in the visual and proprioceptive maps. The model generates from these inputs an arm movement in the spinal map. The basal unit then evaluates a prospective efficiency of the movement, i.e. it tries to guess whether or not the movement will move 


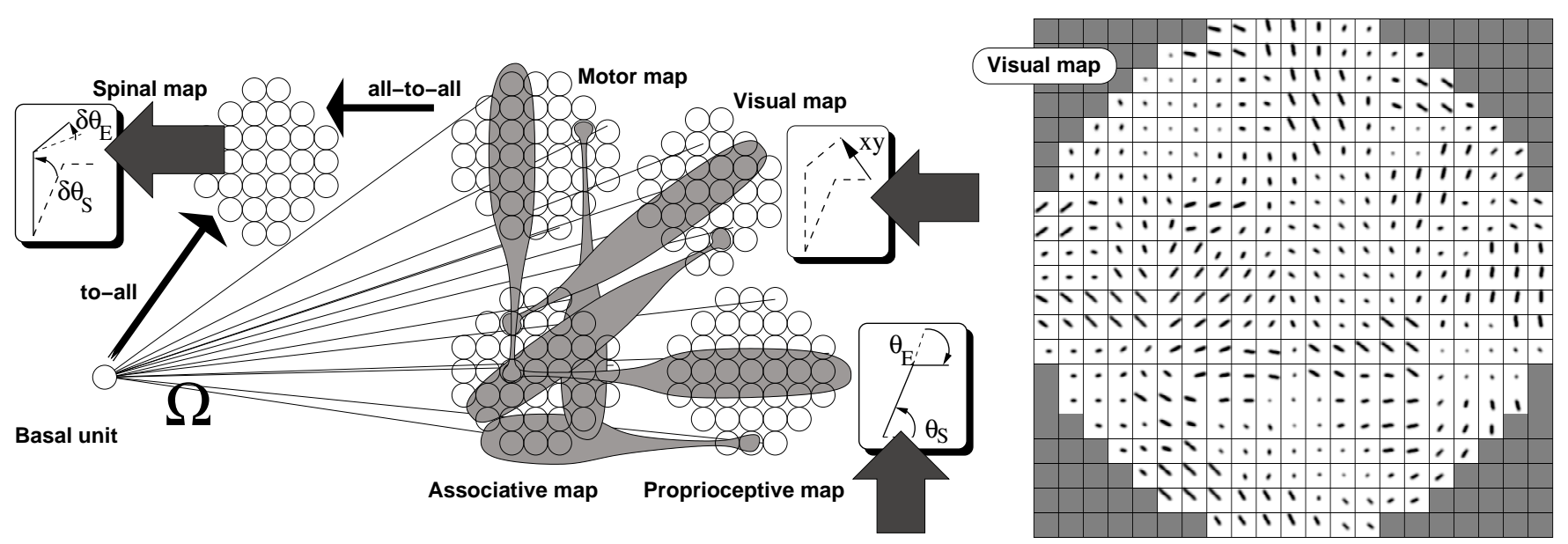

Fig. 6. Visual and proprioceptive input maps are linked, together with a motor map, to an associative map. These links are actually modular stripes. The motor map is the only cortical map directly linked to the reward system: The spinal map is completely linked to the motor map. The basal unit takes its input in all cortical maps, and its output is used to modulate $A^{g}$ activity in the spinal map. The arm posture is represented by the shoulder and elbow angles $\theta_{S}$ and $\theta_{E}$, the visual demand is represented by a $(x, y)$ Cartesian vector and the motor action is a change of the arm angles $\delta \theta_{S}$ and $\delta \theta_{E}$.

the hand to the target. If this evaluation is positive, the computed movement is performed. If not, this decision is blurred in the spinal map by the basal unit so that a random motion is preferred. After the movement has occurred, its actual efficiency is determined, and the basal unit corrects its evaluation, if needed.

Our model provides action reward in a very crude way, and, while the reasons for its existence are biologically-inspired, it does not try to emulate the biological reward system that is believed to exist in the brain, and more specifically in the basal ganglia (see Schultz, 1997). It does, however, mimic its basic functional principles. The simple reward implemented in our model actually estimates the performed action's efficiency (policy evaluation) and compares its evaluation with the actual efficiency. It is the result of this comparison that biases activation in our model's spinal map, so that future actions provide more reward (policy improvement). Model architecture ensures that only a small part of the model (the basal unit and the spinal map), standing at motor level, deals with the rewarding process. Let us now consider in detail the basal unit and the spinal map.

The basal unit's role is to predict the efficiency of the model in its current condition. It considers the $A^{\star}$ activity pattern on all the maps except the spinal one, and computes the prospective efficiency $E_{p}$ from weighted links, with weight vector $\Omega$, to the map units according to equation 3 . That prospective efficiency is compared to the real efficiency $E_{r}$ when a movement is performed. $\mu$ is a learning coefficient.

$$
E_{p}=\prod_{\text {maps }} \sum_{\text {units } \in \text { map }} \omega_{i} A_{i}^{\star}, \Delta \omega_{i}=\mu\left(E_{r}-E_{p}\right) \times A_{i}^{\star}
$$

Thus, the estimated efficiency comes closer to the real efficiency. If the predicted efficiency $E_{p}$ is too low, spinal activity becomes random, and the spinal map performs a random action. If $E_{p}$ is high enough, however, the spinal map computes its action from its cortical input provided by the motor map. As was previously mentioned, each unit in the spinal map is related to a specific movement, and the movement that is actually performed corresponds to the unit with the highest activity. The other difference between the spinal map and other Bijama maps is that its cortical connections, that are from the motor map, form an all-to-all connectivity, in order to ensure that the fixed action distribution in the spinal map does not disable the plasticity of the motor map organization.

\section{Results}

When the model has learned during enough time steps, all of the cortical modules are organized. Their organization is the one that allows coherence to occur as the model processes its different inputs, while remaining continuous when possible, so that close units in the map have close prototypes. Discontinuities may appear due to the constraints imposed by the other maps via the stripe connections (see. fig. 6-right). This resembles observations of discontinuities in V1, that have been shown by the RF-LISSOM model by Miikkulainen, Bednar, Choe, \& Sirosh, 1997. In that work, discontinuities were allowed by the learning of lateral influences. On the contrary, here, the lateral influences have no plasticity, and it can be suggested that discontinuities are the result of multi-map joint organization. This raises a discussion about the nature and the function of such discontinuities, that is out of the scope of this paper, and that will be more relevant when future versions of the Bijama model will allow lateral plasticity, as the RFLISSOM model does.

The model computes a reward each time it performs an action. That reward is a measure (in $[0,1]$ ) of how close the final arm position (after the movement) is to the target position. It increases (cf. fig. 7) as the model learns what actions provide the best reward. The resulting distribution in the visual map shows unbiased direction (cf. fig. 6-right), meaning that 


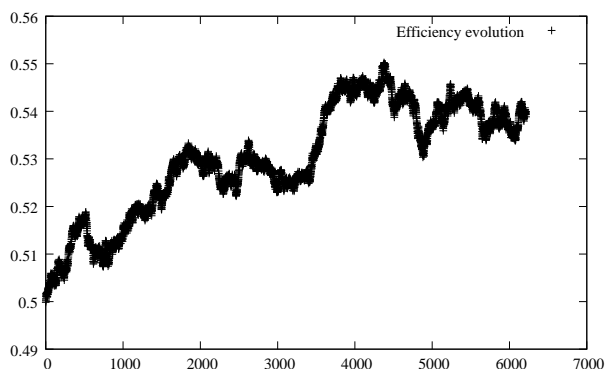

Fig. 7. Reward evolution: Reward is low-pass filtered and a time unit corresponds to 100 time steps

all possible targets have been considered by the model. The important point here is that such a perceptual organization is related to the use of reward, even if reward is only presented to motor part of the model. Here again, joint organization has kept coherence, avoiding inclusion of explicit rewarded related mechanisms at purely perceptual parts of the architecture.

\section{Conclusions}

The model presented in this paper is a computational instantiation of some cortical paradigms. It models cortical computation as a process that scatters information in many modal areas while keeping the whole coherent. The solution to that problem proposed by the Bijama model relies on both a robust competition mechanism and the setting up of some modular stripes that connect self-organizing modules. The resulting dynamics is a multi-modal relaxation process that captures relations between the modalities computed in each module. The genericity of the model has been tested on two distinct problems. In the arm-reaching problem, the need of rewarded learning has been introduced, but the architecture is able to keep the cortical parts of the model homogeneous by allowing reward computation at non cortical motor parts only. The first encouraging experimental results presented here promote the Bijama model as a good candidate for designing self-organizing processes in full behavioral architectures, whereas current self-organizing architectures often concern the mapping of mono-modal distributions at low level filtering stage only. Further work will investigate time processing with such an approach since it is mandatory for any realistic close loop control. Moreover, including time in the Bijama model, that has already the ability to deal with reward, aims at addressing planning of actions with a neural framework, and may lead as a consequence to a computational modeling of prefrontal cortex. The challenge will be then to rely on Bijama to propose an unified view of cortical processing since current models of prefrontal and posterior cortex are based on quite different paradigms.

The authors would like to thank the Lorraine region, the Robea program of the CNRS and the European MirrorBot project for their support.

\section{REFERENCES}

Alexandre, F., Guyot, F., Haton, J.-P., \& Burnod, Y. (1991). The Cortical Column: A New Processing Unit for Multilayered Networks. Neural Networks, 4, 15-25.

Amari, S.-I. (1977). Dynamical study of formation of cortical maps. Biological Cybernetics, 27, 77-87.

Burnod, Y. (1989). An adaptive neural network : the cerebral cortex. Masson.

Doya, K. (1999). What are the computations in the cerebellum, the basal ganglia, and the cerebral cortex ? Neural Networks, 12, 961-974.

Frezza-Buet, H., \& Alexandre, F. (2002). From a biological to a computational model for the autonomous behavior of an animat. Information Sciences, 144(1-4), 1-43.

Grossberg, S. (1976). Adaptative pattern classification and universal recoding, i:parallel development and coding of neural feature detectors. Biol. Cybernetics, 23, 121-134.

Guigon, E. (2003). Computing with populations of monotonically tuned neurons. Neural Computation, 15(9), 2115-2127.

Hubel, D. (1995). Eye, brain, and vision. W H Freeman.

Kohonen, T. (1988). Self-organization and associative memory. Springer-Verlag.

Martinez, T., Ritter, H., \& Schulten, K. (1990). Threedimensional neural net for learning visuomotor-coordination of a robot arm. IEEE Transactions on Neural Networks, 1, 131-136.

Menard, O., \& Frezza-Buet, H. (2003). Multi-map selforganization for sensorimotor learning: a cortical approach. In Proc. international joint conference on neural networks.

Menard, O., \& Frezza-Buet, H. (2004). Rewarded multi-modal neuronal self-organization: Example of the arm reaching movement. In Proc. international conference on advances in intelligent systems theory and application.

Menard, O., \& Frezza-Buet, H. (2005). Coherent learning in cortical maps: A generic approach. In Proc. international joint conference on neural networks.

Menard, O., Frezza-Buet, H., \& Alexandre, F. (2004). Multicriteria self-organization: Example of motor-dependant phonetic representation for a multi-modal robot. In Neurobotics workshop, 27th german conference on artificial intelligence.

Miikkulainen, R., Bednar, J., Choe, T., \& Sirosh, J. (1997). Self-organization, plasticity, and low-level visual phenomena in a laterally connected map model of the primary visual cortex. In P. S. R.L. Goldstone \& D. Medin (Eds.), Psychology of learning and motivation (pp. 257-308).

Miller, K., Pinto, D., \& Simons, D. (2001). Processing in layer 4 of the neocortical circuit: new insights from visual and somatosensory cortex. Current Opinion in Neurobiology, 11, 488-497.

Pulvermüller, F. (2003). The neuroscience of language. Cambridge University Press.

Schultz, W. (1997). Dopamine neurons and their role in reward mechanisms. Current Opinion in Neurobiology, 7, 191-197.

Taylor, J. (1997). Neural networks for consciousness. Neural Networks, 10(7), 1207-1225. 